\title{
Mobile Computing with MPEG-21
}

\author{
Marios C. Angelides ${ }^{1}$, Anastasis A. Sofokleous ${ }^{1}$, and Christos N. Schizas ${ }^{2}$ \\ ${ }^{1}$ Brunel University, Uxbridge, Middlesex, UB8 3PH, UK \\ \{marios.angelides, anastasis.sofokleous\} @brunel.ac.uk \\ ${ }^{2}$ University of Cyprus, Nicosia, Cyprus \\ schizas@ucy.ac.cy
}

\begin{abstract}
In the field of multimedia content provision, many researchers investigate numerous techniques for adapting the content based on device, network, environment and user characteristics. In this paper, we present an MPEG-21 middleware design for dynamic content adaptation and we demonstrate a true mobile multimedia client that is able to communicate information about device and network characteristics and provide the necessary tools for the configuration of user preferences.
\end{abstract}

\section{Introduction}

MPEG-21 aims at understanding how the various elements of the infrastructure for the deployment of multimedia applications fit together. Various terms and definitions have been defined in this framework, such as content which have been replaced by the term Digital Item. Digital item adaptation is defining the personalization of the selection and delivery of multimedia content to the individual users. Content adaptation is necessary so as to make the content universally accessible (Universal Multimedia Access) since different networks and devices imply different quality of service, bit rate, computing and presentation capabilities and also different users imply different preferences (e.g. content type, content quality, etc) and usage history[1],[2]. However, MPEG-21 provides only some of the tools necessary to support resource adaptation, descriptor adaptation and QoS management.

Not for all the content types the adaptation operations are the same. For instance, while video adaptation include operations such as transcoding, video summarization, replacement, synthesis [3], for audio the operations are specified as channelDropping and scalableAudio [4]. Using each one of these operations, a digital item will provide different results which will certainly have different satisfaction for each user based on his/her preference and his/her current activities or location. However, the objective usually is not just to adapt the digital item but to provide a result which will be optimized for the network, fit to device characteristics but also have the best satisfaction for the end-user. Therefore the selection of either a single or a combination of operations requires a number of algorithms for determining the operation and adapting the digital item and evaluating the result quality. While numerous types of terminal and mobile devices are being used for content retrieval, currently users' concerns lie not with the end-system, but with the information and information quality[5]. 
In this paper, we present how the MPEG-21 framework will assist in content adaptation and we propose an MPEG-21 middleware design. In addition we present a true multimedia client for mobile devices that uses the current MPEG-21 framework for acquiring the dynamically adapted content. Section 2 discusses the research overview in this area while section 3 presents the middleware design and illustrates the multimedia mobile client. Finally, the paper concludes and presents future work.

\section{Research Overview}

During dynamic adaptation, a system, which is responsible for the whole processing, has to reflect on the adaptation operation characteristics and also the content, user, environment, network and device characteristics. In addition, the delivery of the content, which is mostly depended on the adaptation result, invokes the Quality of Service, a value that is not easy to determine automatically. Having this in mind, it is obvious why until now, in most of the approaches, content adaptation process is a communication handshake calling for a user to select most of the attributes (e.g. quality, format of the content) from an inadequate predefined number of choices. A concise outline of the current research work is discussed below.

In [6], a three-tier architecture was implemented for selecting, adapting and delivering personalized and summarized content to the end users. The media middleware consists of the personalization engine and adaptation engine. In this work, personalization is focused on the insertion or deletion of each video shot depending on user preferences. It is not quite clear how the adaptation engine is achieving the optimal adaptation, but as it is cited, part of the algorithm extracts audiovisual segments from shot boundaries and combines them based on the personalization selection. Moving from ad-hoc content adaptation to a most intelligent adaptation, it is necessary to define the relations of video entity, adaptation, resource and utility [7]. In [7] as in [3] as well, the authors used the term "adaptation operation, resource and utility (ARU) spaces" in order to emphasize the multidimensional constrained problem during the adaptation process. To be more precise, given a particular content unit, adaptation operation space relates to the existence of many adaptation methods that can be applied, such as transcoding, summarization, etc. Furthermore, resource space is defined by the multiplicity of the device and network characteristics related with the content delivery and consumption, such as bandwidth, device computational capabilities, display dimensions, etc. The utility space is quite nearer to the quality of service concept, as it is measuring in its multiple dimension space the user's likings and preferences. Therefore, a point at adaptation space (e.g. corresponded by a defined adaptation operation such transcoding) is associated with' specific resources and utility values which are represented by corresponding points in the resource space and the utility space respectively. Although the above seem to formulate a complete framework for dynamic adaptation, however, during dynamic adaptation, the main problem lies with the fact that the utility value can not be easily measured. Therefore, for a given resource-constrained utility maximization optimization problem, the result of the adapted content can not be easily evaluated without the human factor. In addition, during the searching for the appropriate adaptation operation and its parameters, it is very difficult to know the result of each selection without exercising it at that point of time. 


\section{Design Overview}

The collection, preparation, delivery and consumption chain of content (or digital item) is very long and ambiguous process that begins with the author and usually ends up at the user. A number of actors are involved in the process such as the author, the publisher, the content provider, the content consumer. In addition, many factors and parameters affect that chain, such as the network environment, natural environment, device and user. Whatever device is used (e.g. a normal PC, a mobile phone, a pocket pc, a handheld device, etc) the content must be able to plug-and-play on that device. Therefore, the specific device characteristics must been collected and send for tuning the content according to those characteristics. Usually to the responsible party for this kind of processing is middleware.

Applications supporting MPEG-21 consist of many application layers. Each layer is responsible for a specific functionality, and communication among the layers is achieved through exchange of messages between neighbor layers. For instance, validation for the XML schema or documents would be made by an XML parser. Basic functionality, that is required for each common MPEG-21 tool include mechanisms for search, updating and insertion. Using the basic functionality, the business process may be customized for each application based on its specific requirements. Finally, an application (graphical or console) user interface may be placed on top of the architecture, e.g. a customer user interface for the client, an administration interface for the server, or an application interface providing connection with external systems. The MPEG-21 middleware involves the interaction of many crucial processes, algorithms and operators (e.g. selection[9], adaptation process, adaptation operators, database utilities, content analysis, content evaluation process) but also requires the involvement of MPEG-21 schema and xml readers \& writers. The MPEG-21 framework proposes that during digital item consumption, content adaptation can be utilized through a resource adaptation engine and/or a descriptor adaptation engine [10], [11].

Figure 1 illustrates a likely design of the content adaptation process. In a typical scenario, a user requests a multimedia object (digital item). In the particular case scenario, the digital item is MPEG-7 defined, thus we assume that the annotation step has already taken place. A system (e.g. content provider system) should offer the item to the user in the appropriate format. Specifically, the item has to be adapted for fitting into device, network and environment characteristics, such as terminal display's dimension, bandwidth limitations, etc. While, the information of the digital item is retrieved from the MPEG-21 schema, the information for the terminal and user's preferences is retrieved in a MPEG-21 format from a storage location (e.g. profile storage manager). A number of available adaptation operations assist in the adaptation process. However, the main issue here is what adaptation operation is more suitable to be used and in addition what parameters should be utilized with the particular operation. For that reason, a selection process selects and subsequently tunes the most appropriate adaptation operation with the aid of utilities and several types of information. Among these utilities are modules responsible for content and usage analysis and user-centered generated content evaluation. 


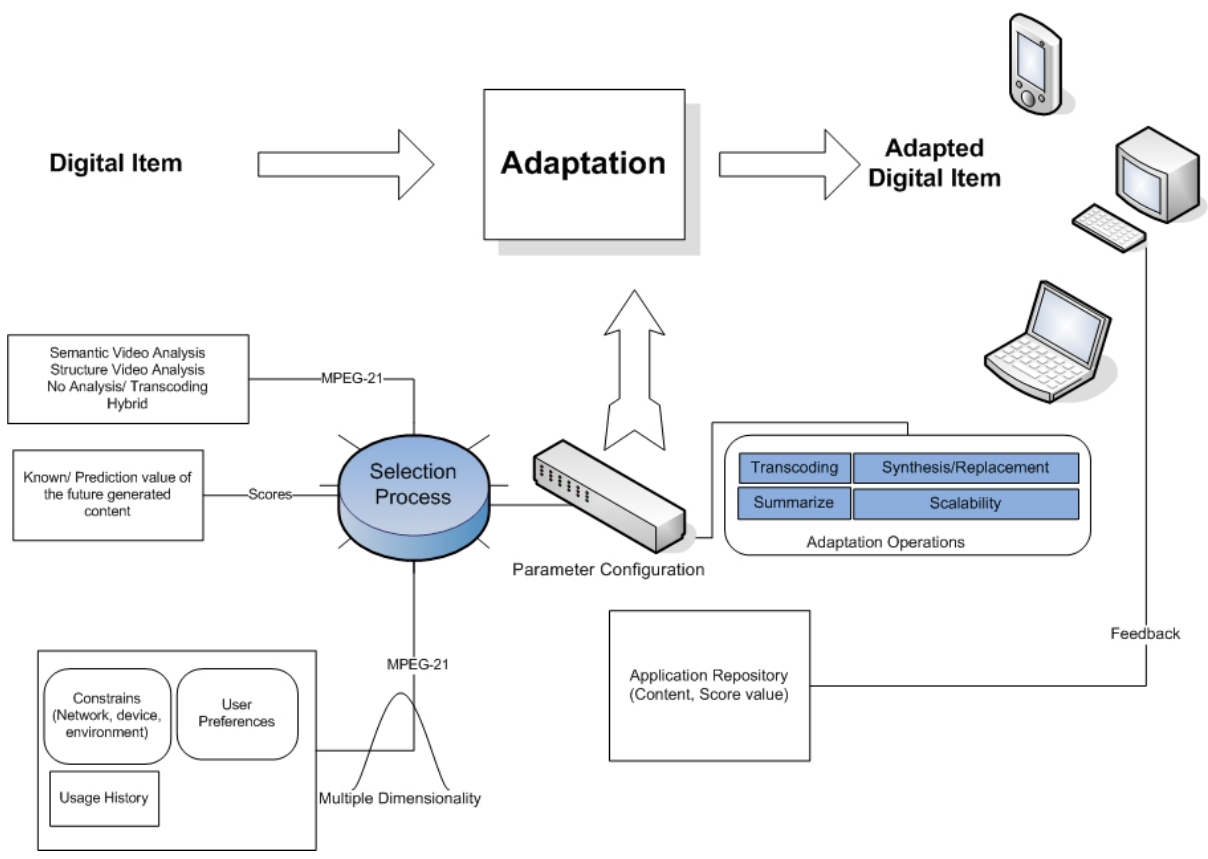

Fig. 1. MPEG-21 Design

Dynamic digital adaptation, which is a module of the middleware and will mostly include optimization algorithms, is designed to interface with the top layer of the MPEG-21 middleware in order to request, adapt, store and provide a digital item along with its descriptors. MPEG-21 middleware is currently under development based on the above design. The following section discusses implementation of the MPEG-21 middleware.

\subsection{The MPEG-21 Middleware Implementation}

The multimedia content adaptation under the MPEG-21 framework is utilized after the digital item request. The Digital Item Upload is part of the content management process followed by each author or publisher. During that phase, a video accompanied by a MPEG-7 file is uploaded to the server. The MPEG-7 file describes the video file syntactically and semantically. For instance, a sport video clip, being stored in MPEG-4 video format, illustrates a soccer player kicking the ball. Therefore, the MPEG-7 file includes the video format characteristics, the description about this event (e.g. date, time, occasion, location, player name, and playing field name), description about the objects of this video clip and their actions, etc. We have developed a web user interface connected with the middleware. Through that, a user can upload digital items (e.g. a video, an image, a music file) with the MPEG-7 file. The MPEG-7 is linked with the digital item and stored in a database.

On the other side, a user requests to watch the particular video clip using his/her mobile device (Digital Item Request). However, while he is in a hurry, he requests 
that he would see the video in black and white color. In addition his device only supports MPEG-2 video and is connected into the internet with GPRS over GSM. Therefore, a MPEG-21 file (constructed at the user site) is sent to the middleware providing the current characteristics of the particular transaction (e.g. his preferences, his devices characteristics, and the network characteristics). The multimedia mobile client is explained in the next section.

The middleware receives the MPEG-21 file(s) and using this file as a parameter calls a selection process. The selection process loads all the information about the user using the MPEG-21 files and the already stored information. User's MPEG-21 characteristics along with his/her usage history data are loaded. The selection process aims to identify the appropriate adaptation operation and therefore to make an appropriate decision on how to adapt an input bit-stream based on many input constrains and characteristics. This kind of decision-taking is characterized as a constrained optimization problem involving algebraic variables that represent adaptation parameters, media characteristics, usage environment inputs, or any combinations thereof [9]. The solution, yielding the decision, can then be computed by a universal process independent of what the variables represent. Analyzing the MPEG7 file and the MPEG-21 file, the selection process has to search for an adaptation operation that will transform the MPEG-4 color video to the MPEG-2 black/white video. The transcoding operation is selected and is utilized (Digital Item Adaptation).

The middleware is streaming the on-air adapted digital item (Digital item provision). The mobile device receives the streaming video and display the black/white video to the user (Digital item consuming). The user can evaluate the content using his device browser (explained also in the next section). End-user devices must be able to interact with the middleware infrastructure so as to provide the necessary information (e.g. characteristics and user' preferences). The interaction will guide the adaptation process. In addition a digital item viewer is needed fore viewing adapted items. The following section presents a mobile application, called MPEG-21 mobile client, which consists of an interactive MPEG-21 browser and a digital item viewer.

\subsection{MPEG-21 Mobile Client}

The proposed system consists of a digital item Viewer, a sub-system mainly designed and developed for mobile devices, and a MPEG-21 interactive browser (figure 2). Both of the sub-systems are implemented in J2ME (java for mobile devices) and a number of extra J2ME libraries which come with mobile phones (e.g. MMPAPI). Thus, the MPEG-21 mobile client is able to run on any mobile device. Using this integrated and complete mobile system, a user may specify his/her preferences under the MPEG-21 framework and therefore configure digital items according to User preferences[12], request adaptation for resources, and view the desired digital item adapted by the MPEG-21 middleware.

MPEG-21 Interactive Browser communicates directly with the MPEG-21 middleware and provides the necessary tools and specifications for both user and device total interaction under the MPEG-21 standard. The expandable browser comprising many components, enhances the optimization of the content adaptation process, since during communication with the middleware, it automatically provides information about the mobile device and network characteristics. 
Many user preferences (e.g. content, video and audio presentation, color, presentation priority, etc), which also are proposed by the MPEG-21 experts, shape a user profile and therefore guide content adaptation. When a person uses a different mobile device or terminal, his/her preferences may not change. That kind of action requires having user's profile portable, which implies either a number of algorithms for moving intelligently the profile each time a user change a device or just to have each user's profile at a central online server. In addition, with regards to customizing preferences for each device, there will be few differences to opting for a fresh specification. The user profile may be saved in repositories[13] or in databases[14]. In DIA, session mobility refers to the transfer of configuration - state information that pertains to the consumption of a Digital Item on one device to a second device[15].

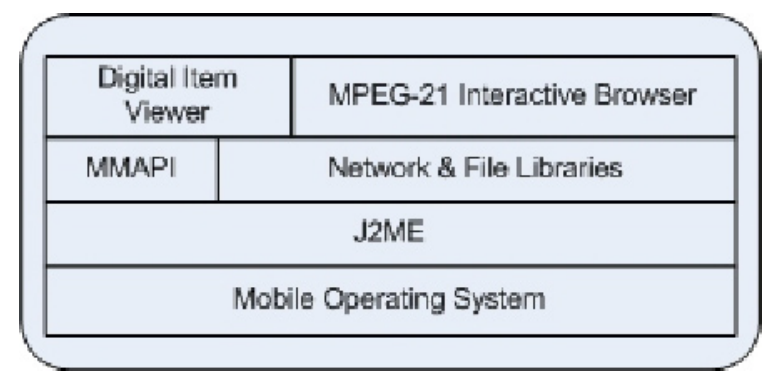

Fig. 2. The Multimedia Mobile Client
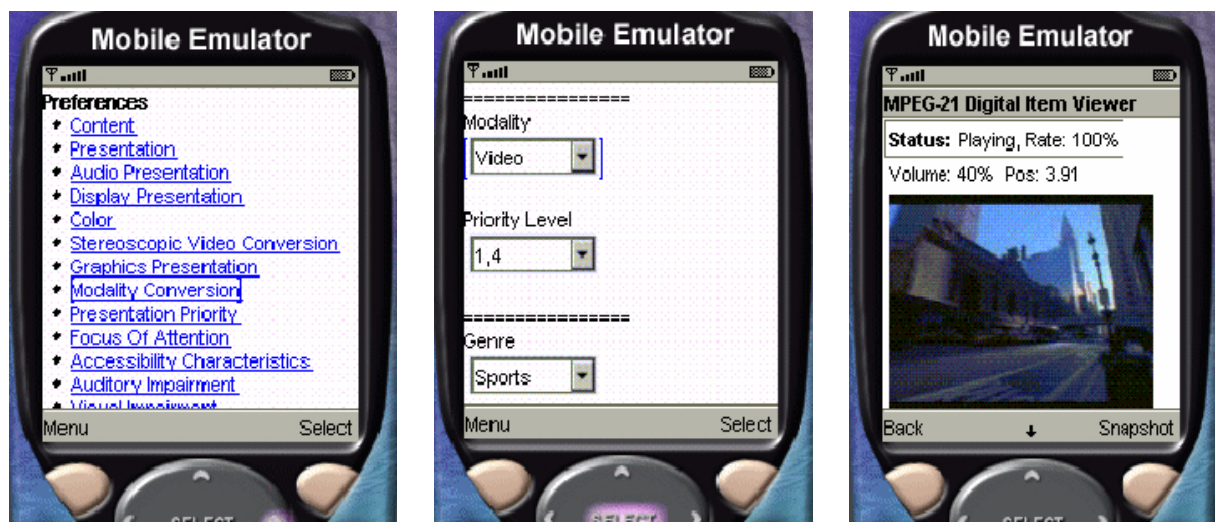

Fig. 3. (a) MPEG-21 User Preferences. (b) Modality Priority. (c) MPEG-21 Digital Item Viewer.

The MPEG-21 interactive browser identifies the user, and loads his/her preferences during initialization. Furthermore, a user is able to update his/her preferences at any time (figure 3a). User characteristics include general user information, content preferences, presentation preferences, accessibility characteristics, mobility characteristics and destination [16]. Currently most of these have to be inserted manually since most of the preferences cannot be extracted anywhere else but from 
the user. User location can be determined and inserted automatically by the system. The interactive browser can be easily expanded and modified to support location services so as to provide the location information to MPEG-21 middleware. Therefore, adaptation algorithms will use this kind of information in their calculations for better digital item adaptation since specific preferences usually vary based on location. For instance, users would like to watch high quality movies at home even when there is a request to watch in black and white rather than color.

Measuring video quality or image quality is a complex process, even with the aid of human factor, since quality has not been defined effectively for dynamic environments. Approaches for quality evaluation are using the objective quality, which requires a number of computing algorithms, or the subjective quality, which requires manual effort [17]. In some cases a user or the system may specify quality threshold under which it is declared that no acceptable experience can be provided. However providers shall always deliver the best possible experience [8]. A user may use the mobile interactive browser for evaluating the adapted content by updating the AdaptationQoS parameters (indirectly). Quality of service is defined under MPEG-21 as AdaptationQoS. It describes the relationship between constraints, possible adaptations, and qualities in order to support media resource adaptation for terminal and network quality of service [4]. The AdaptationQoS descriptor provides the means to trade-off these parameters with respect to quality so that an adaptation strategy can be formulated and optimal adaptation decisions can be made in constrained environments. For example, a user can define the relative order of each conversion of an original modality and the numeric weight of each conversion (figure $3 \mathrm{~b}$ ). The weights of conversions with the Adaptation $Q o S$ parameters will help the selection process to determine when conversion should be made. The Mobile digital item viewer presents adapted content to users. Currently, the viewer supports audio, video, image and text delivery over http, https, rtp, rstp. For instance, a user can watch a movie as streaming video via the rstp protocol or can download it and watch it via the http protocol (figure $3 \mathrm{c}$ ).

While a part of MPEG-21 framework is about user, terminal, network, usage, natural environment characteristics, it is unclear how these fit to a practical scenario or who and how is providing that information. These characteristics require specific treatment by specific actors, such as a mobile device, a user, or middleware. A user has to insert most of their data manually but the implementation of intelligent algorithms on a device or a middleware will automate this. The browser is able to retrieve device characteristics (e.g. device type, operating system display resolution, codec and content types supported by devices, processor, memory, etc) and use this information during digital item adaptation. Network characteristics can also be defined by the user manually or automatically by the device and middleware. The collaboration model, which illustrates the sources of "MPEG-21 characteristics", is illustrated in figure $4 \mathrm{a}$.

Figure $4 \mathrm{~b}$ shows priority preference for general resources, where a user wishes to have high video QoS by assigning a priorityLevel of 1.5 to video resources. The user is also interested in Sports and gives a priorityLevel of 1.6 to this genre. So, the resources of video modality and Sports genre, especially the Sport videos, should have better qualities after adaptation. Note that the user already knows the default priorityLevel of resources is 1.0. However, the same user would specify different 
weight values under different circumstances e.g. when he is in his/her car and he wants the content in less elapsed time. The priorityLevel is defined within user characteristics and is filled manually by a user (figure $4 a$, figure $3 b$ ).

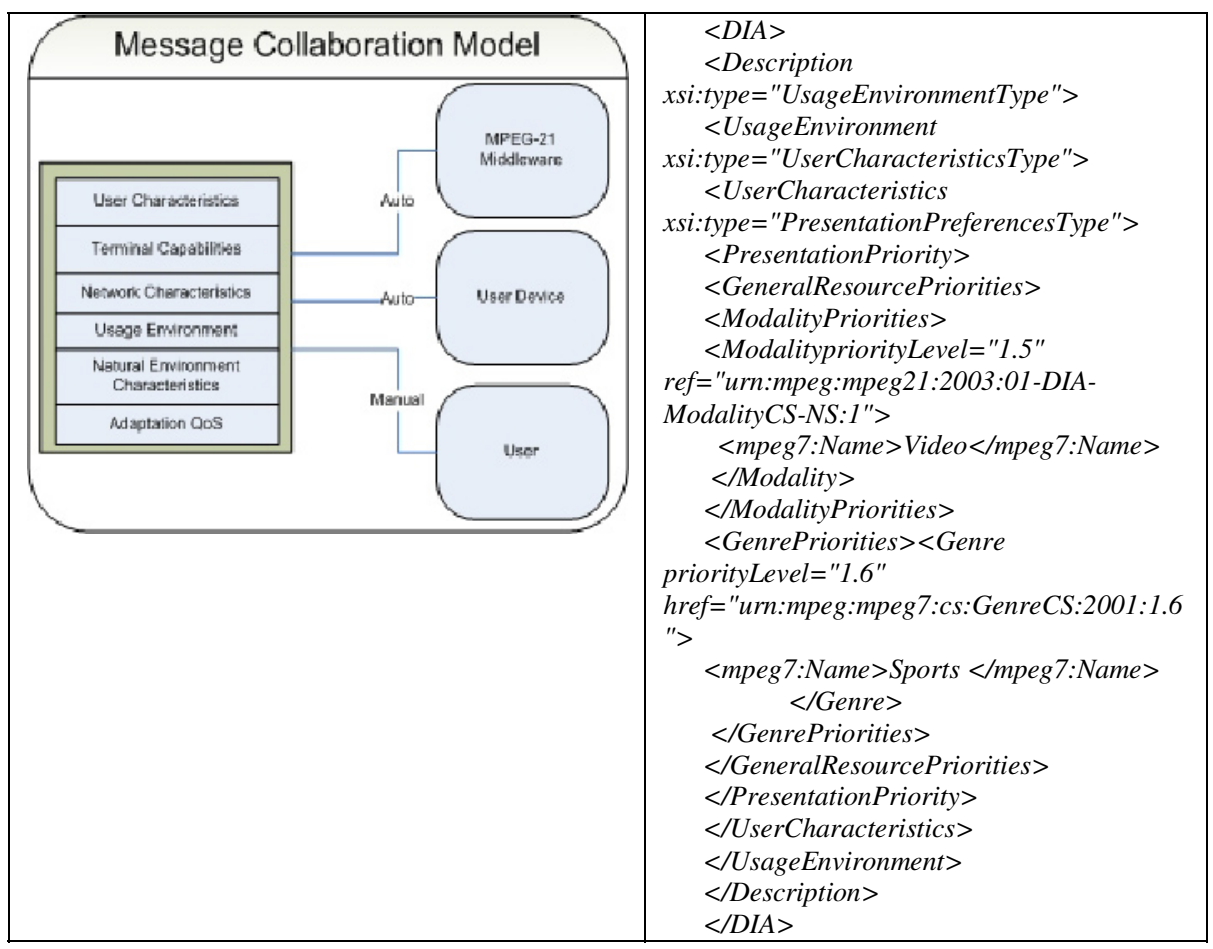

Fig. 4. a) Automatic and manual gathering of characteristics. b) MPEG-21 Presentation Priority.

\section{Performance Evaluation and Complexity}

During the performance evaluation of the architecture, a series of tests were performed on sample of user requests. A number of mobile phones having the multimedia mobile client were generating requests. Benchmarks revealed that the middleware performance is depended on the selection process and the selected adaptation operator. The selection process is heuristic, which makes it very difficult to determine and characterize precisely the execution time. Whilst each adaptation operator performance is different, it can be calculated. The main factors that affect each adaptation operator performance are the size of the digital item (e.g. video size), the initial and target format type and characteristics. The server's configuration plays an important role for an adaptation operator (e.g. a dual 2,4 $\mathrm{GHz}$ CPU with two simultaneously real time transcoding channels may assist the transcoding operator to exceed real time). Most of the adaptation operation results can be predicted. For instance, consider the case of a mobile client which requests a video movie but the mobile device does not support the video format. Therefore, the 
selection process finds an adaptation operation that can export to audio with parameters such as 8-bit/16-bit/32-bit sample type b. stereo/mono. These parameters may play an important role to the size of the adapted audio (figure 5). The final size can be calculated before adaptation takes place. However, some characteristics of the final digital item cannot be determined, such as the measurement of video, image or audio quality.

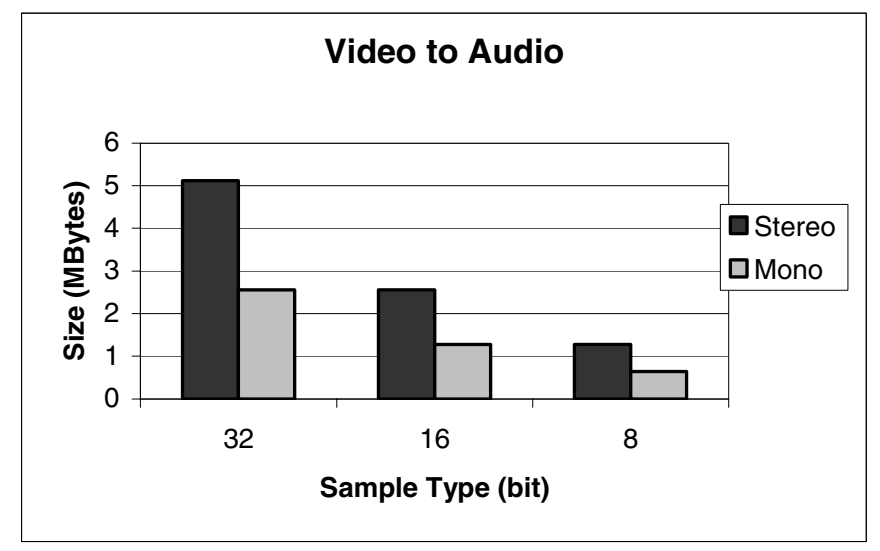

Fig. 5. Video (MPEG-2) to Audio adaptation

\section{Conclusions and Future Work}

In this paper we exploit how mobile computing can use MPEG-21 by illustrating a design of MPEG-21 middleware and a prototype multimedia mobile client. MPEG21 mobile client allows a user to define his/her preferences and at the same time include mechanisms for retrieving and sending device and network information to the middleware. The middleware is designed to provide content satisfaction for most user preferences and device and network constrains. We are currently implementing the MPEG-21 middleware and its algorithms (adaptation, selector and evaluation algorithms).

\section{References}

1. ISO/IEC, MPEG-21 Digital Item Adaptation, N5845, 2003

2. Burnett, I., Walle, R. V., Hill, K., Bormans, J.,Pereira, F.: MPEG-21: Goals and Achievements. IEEE Multimedia, Vol.10(4). (2003) 60-70

3. Chang, S. F.,Vetro, A.: Video Adaptation: Concepts, Technologies, and Open Issues. Proceedings of the IEEE, Vol.93(1). (2005) 148-158

4. Feiten, B., Wolf, I., Oh, E., Seo, J.,Kim, H.-K.: Audio Adaptation According to Usage Environment and Perceptual Quality Metrics. IEEE TRANSACTIONS ON MULTIMEDIA, Vol.7(3). (2005) 446-453

5. Ramesh, J.: A True Multimedia Client. Multimedia IEEE, Vol.12(2). (2005) 103-104 
6. Tseng, L., Ching-Yung, L.,Smith, J., R.: Using MPEG-7 and MPEG-21 for Personalizing Video. IEEE Multimedia, Vol.11(1). (2004) 42-52

7. Kim, J.-G., Wang, Y.,Chang, S.-F.: Content-Adaptive Utility-Based Video Adaptation. Proceedings of IEEE Int'l Conference on Multimedia \& Expo, Vol.3 (2003) 281-284

8. Pereira, F.,Burnett, I.: Universal Multimedia Experiences for Tomorrow. IEEE Signal Processing Magazine, Vol.20(2). (2003) 63-73

9. Mukherjee, D., Delfosse, E., Kim, J.-G.,Wang, Y.: Optimal Adaptation Decision-Taking for Terminal and Network Quality-of-Service. IEEE TRANSACTIONS ON MULTIMEDIA, Vol.7(3). (2005) 454-462

10. Timmerer, C.,Hellwagner, H.: Interoperable Adaptive Multimedia Communication. IEEE Multimedia, Vol.12(1). (2005) 74-79

11. Panis, G., Hutter, A., Heuer, J., Hellwagner, H., Kosch, H., Timmerer, C., Devillers, S.,Amielh, M.: Bitstream Syntax Description: A Tool for Multimedia Resource Adaptation within MPEG-21. EURASIP Signal Processing: Image Communication Journal, Vol.18(8). (2003) 721-747

12. De Keukelaere, F., De Zutter, S.,Van de Walle, R.: MPEG-21 Digital Item Processing. IEEE TRANSACTIONS ON MULTIMEDIA, Vol.7(3). (2005) 427-434

13. Jerez, H., N., Liu, X., Hochstenbach, P., Van de Sompel, H.: The Multi-faceted Use of the OAI-PMH in the LANL Repository. Proceedings of the 4th ACM/IEEE-CS joint conference on Digital libraries, (2004) 11 - 20

14. Kosch, H.: Distributed Multimedia Database Technologies Supported by MPEG-7 and MPEG-21. 1st edn. CRC PRESS, New York (2004)

15. Vetro, A.,Timmerer, C.: Digital Item Adaptation: Overview of Standardization and Research Activities. IEEE TRANSACTIONS ON MULTIMEDIA, Vol.7(3). (2005) 418-416

16. Vetro, A.: MPEG-21 Digital Item Adaptation: Enabling Universal Multimedia Access. IEEE Multimedia, Vol.11(1). (2004) 84-87

17. Richardson, I. E. G.: H.264 and MPEG-4 Video Compression: Video Coding for Nextgeneration Multimedia. 1st edn. John Wiley, Chichester (2003) 